\title{
The Mesoscale Numerical Simulation of the Effectiveness of an Artificial Precipitation Enhancement Operation
}

\author{
J. Wang, Y.W. Chen \\ Jiangsu Climate Centre, China
}

\begin{abstract}
According to the mechanism and functions of the AgI seeding, the AgI seeding course was coupled into the operational mesoscale Weather Research and Forecasting(WRF) mode with reasonable boundary and initial conditions. Then the model is applied to analyse convective artificial precipitation enhancement over the Tian Mu Lake at Li Yang for the improvement of the ecological environment. The results from analysis of numerical simulation show that after seeding the strong echo area of cloud enlarges, the average echo intention increases significantly and the rainfall on the ground increases. The microphysical analysis show that after seeding the content of the ice, snow and grauple increases, correspondingly the content of the rain water in the low-level increases as well, which results in the enhancement of the precipitation.
\end{abstract}

Keywords-AgI seeding; the effectiveness of the precipitation enhancement; mesoscale numerical simulation; the ecological environment

\section{INTRODUCTION}

The assessment and test of the effectiveness of the rain enhancement by artificial means is the key issue of the research and operation of the weather modification. The integrative test methods of the effectiveness of the rain enhancement by artificial means include the statistic, physical and numerical simulation tests. The numerical simulation method is well content with the fundamental requirement of the objection, repeating and forecasting in the assessment of the effectiveness of weather modification. By now the 3dimension convective cloud models constructed by Kong Fang-you and Hu Zhi-jing is mainly adopted to the numerical simulation test of the effectiveness of the convective cloud rain enhancement. However, the imperfection of treating with the initial and boundary conditions induce to inability of simulation the complex phenomenon of the real atmosphere. The cloud models compute in the simple boundary condition mostly, and the boundary condition of the underlying surface is assumed uniformly. Therefore, the adoption of the mesoscale operation model with the perfect initial and boundary condition in the analysis by the seeding numerical simulation provides a new method for the test and forecast in quantity of the effectiveness of the regional rain enhancement from convective cloud. According to the mechanism of AgI seeding and effect, the new unhydrostatic mesoscale WRF model coupled with the AgI seeding course is applied to the analysis of the effectiveness of the artificial rain enhancement in Tianmu Lake in Liyang on August 28th, 2007 by mesoscale seeding numerical simulation.

\section{Method OF THE SEEDING NUMERICAL SimUlation}

According to the literatures [1-3], considering there mechanisms of the growth of the AgI particle in the cloud, which are the nuclearizations from the freezing when the artificial ice nucleus catch the cloud and rain drop and the sublimation when the artificial ice nucleus catch the vapour, the AgI seeding course for rain enhancement is coupled to the WRF model. The expression of the AgI seeding course is as follow:

$$
\begin{aligned}
\frac{d X_{S}}{d t} & =-D_{X S}+\text { Source }+\sin k(1) \\
\sin k & =S_{B C}+S_{I C}+S_{B R}+S_{I R}+S_{D V}(2)
\end{aligned}
$$

$\mathrm{X}_{\mathrm{s}}$ is the proportion content of AgI, Source is the item of the AgI source, namely the item of artificial seeding, Sink is the item of the AgI convergence, $\mathrm{S}_{\mathrm{BC}}, \mathrm{S}_{\mathrm{IC}}, \mathrm{S}_{\mathrm{BR}}, \mathrm{S}_{\mathrm{IR}}, \mathrm{S}_{\mathrm{DV}}$ correspond to the mechanisms of the growth of the AgI particle in the cloud. The vapour sublimate to the ice, the cloud drop freeze to the ice, and the rain drop transform to the frozen drop, which make the density of the ice change:

$$
\begin{gathered}
N N U_{v a i}=S_{d v} \Delta t m_{S}^{-1}(3) \\
N N U_{c a i}=\left(S_{b c}+S_{i c}\right) \frac{N_{a}(\Delta T)}{N_{a}\left(20^{\circ} \mathrm{C}\right)} \Delta t m_{s}^{-1} \\
N N U_{r a i}=\left(S_{b r}+S_{i r}\right) \frac{N_{a}(\Delta T)}{N_{a}\left(20^{\circ} \mathrm{C}\right)} \Delta t m_{s}^{-1}
\end{gathered}
$$

$N_{a}(\Delta T) / N_{a}\left(20^{\circ} \mathrm{C}\right)$ is the proportion of the active catalyser at the over-cool temperature of, $m_{s}$ is the mass of AgI $\left(=2.38 \times 10^{-14} \mathrm{~g}\right), \quad$ is the integral time of the model. Besides, because the WR-98 equipment of the artificial rain enhancement contains the AgI aerosol, so the nucleus ratio of the AgI in the model is the ratio of the BR-91-Yaerosol.

\section{ApPliCAtion OF THE CASE}

Jiangsu Artificial Weather Office held the operation of rain enhancement by artificial means for ecology regulation of water in Tian $\mathrm{Mu}$ Lake in $\mathrm{Li}$ Yang together with the Changzhou Artificial Weather Office on August 28th in 2007. When the drought is alleviated, the amount of the water of the Tian Mu Lake is complemented and the quality of the water is purified by the rain from the nature and artificial enhancement.

\section{A. Analysis by Seeding Numerical Simulation}

According to the method of the seeding numerical simulation introduced in the chapter 2, the operation of 
artificial rain enhancement in Tian Mu Lake at 20:24 is analyzed by seeding numerical simulation with the new mesoscale unhydrostatic WRF model of version 2.1.2 coupled with the course of the AgI seeding. The numerical simulation adopts two-way nest. The outer nest is $101 \times 81$, and the inner nest is $154 \times 112$. The grid distances of the nests are $9 \mathrm{~km}$ and $3 \mathrm{kmseparately.} \mathrm{The} \mathrm{vertical} \mathrm{atmosphere} \mathrm{adopts} 31$ levels. The initial field is obtained by the National Centers for Environmental Prediction(NCEP)/National Oceanic and Atmospheric Administration (NOAA) $1^{\circ} \times 1^{\circ}$ reanalysis data. Otherwise, the numerical simulation adopts the Medium Resolution Forecast (MRF) boundary scheme, Betts-MillerJanjic (BMJ) convective parameterization scheme, Thompson microphysical process scheme, Rapid radiation Transfer Model(RRTM) long-wave radiation and Dudhia short-wave radiation schemes.

Among this seeding numerical simulation, cloud microphysical scheme adopts the Thompson microphysical scheme. This scheme is the correction of the Reisner scheme. Thompson scheme divides water substance into cloud water, cloud ice, rain, snow and grauple, and considers more than 30 microphysical processes, which is the most detailed cloud microphysical scheme in the version 2.1.2 WRF model, and also is the only scheme of forecasting the density of the ice in WRF model. The density of the ice is not abstained by experiential expression, so the computation of the microphysical process is more correct. However, because the scheme does not forecast the density of the cloud water, rain, snow and grauple, it is not perfect contrast to the double parameter microphysical scheme in the convective cloud model. For example, after seeding cold cloud the affection of cloud water, rain, snow, and grauple only reflects at proportion content and does not at the proportion density result from the change of the ice density. But the WRF model can correctly simulate the cloud microphysical developing process and the main change after seeding through the avail of revised Thompson microphysical scheme superior in the trading with the boundary and initial field.

8 WR-98 rockets are shot at this seeding operation. Every rocket contains 10gAgI. The total amount of the seeding AgI is $80 \mathrm{~g}$. According to the operation lifting elevation $\left(60^{\circ}\right)$, the height of the $0^{\circ} \mathrm{C}$ level(about $5 \mathrm{~km}$ ), the figure of the trajectory of the WR-98 rocket, the time of seeding above $0^{\circ} \mathrm{C}$ level is $29 \mathrm{~s}$, and the total time of seeding is $37 \mathrm{~s}$, so the seeding amount of AgI above $0{ }^{\circ} \mathrm{C}$ level is 65g, namely effective seeding amount. The average seeding height is about $5.5 \mathrm{~km}$. When the model integrates to 20:24, the AgI is seeded at the vertical level which is most close to the average height of the seeding ( $\eta 16$ level). The seeding horizontal point is at the center of the severe echo. The 65gAgI is seeded within 8 integral steps. One step is 20s, namely the integral step of the model. The way of the seeding adopts the plane seeding, namely 9-grid seeding within the radius of $3 \mathrm{~km}$ at the center of the severe echo at the level-16(-4 $\left.\sim 5^{\circ} \mathrm{C}\right)$.

After seeding AgI the comparison of the echo reflectivity and the accumulated rainfall on the ground between the nature cloud and the seeding cloud in the operation area show that the change of the accumulated rainfall in the operation area can be mainly divided into two phases. At the first phase (20:30 22:36) the seeding cloud together with the cloud behind the seeding cloud form the Southeast-Northwest echo strip, which develops and strengthens after seeding comparing with the nature cloud, so as to form the Southeast-Northwest increasing rain strip in the operation area. At 20:54 the maximum echo reflectivity of the nature cloud is $35 \sim 40 \mathrm{dBZ}$, whereas the maximum echo reflectivity of the seeding cloud increases by $40 \sim 45 \mathrm{dBZ}$ and the rainfall nearby the center of the severe echo begins to increase. At 21:24 contrary to the nature cloud, the area whose echo reflectivity is greater than $30 \mathrm{dBZ}$ of the seeding cloud enlarges, the average echo reflectivity increases, the intention and range of the increasing rainfall increases, and the maximum of the increasing rainfall reaches $0.7 \mathrm{~mm}$. At 21:54 when the severe echo behind the seeding cloud converges into the echo of the seeding cloud, the echo reflectivity of the seeding cloud is greater than the nature cloud at the center of convergence place, the center of the increasing rain reaches $0.9 \mathrm{~mm}$, and the strips of the increasing rainfall, accumulated rainfall, echo present the Southeast-Northwest distribution, and the decreasing rain area appears locally downriver. After then the echo strip in the operation area moves to the south significantly, the echo reflectivity at the middle and upriver at the front of the center of the heavy rain increases, and the echo reflectivity at the downriver locally decreases. At 22:24 the range of the increasing rain enlarges to the south, the area and intention of the decreasing rain downriver increase, the velocity of the increasing rain all over the operation area becomes slower, and there is the sever echo appears in the Northeast at the center of the heavy rain, which forms the new Southwest-Northeast echo strip together with the echo in the South at the center of the heavy rain. At the front of the second phase (22:42 23:18) the new strip moves to the south gradually (Figure1d, e). At 22:54(Figure1e) the southwest part and northeast back part of the new echo strip is greater than the nature cloud, correspondingly the accumulated rainfall increases slightly. Whereas the northeast frontal part of the new echo strip is weaker than the nature cloud slightly, so that the downriver strip of the increasing rain ruptures. The total strip of the increasing rain presents the South-North distribution. Then at the back of the second phase(23: 24 0:00) the southwest part of the new echo strip has ever passed the operation area, but the frontal and back of the northeast part of the new echo strip breaks up. After breaking (Figure1f) the back echo locates the south of the center of the heavy rain and the frontal echo locates the east of the center of the heavy rain. The back echo is greater than the nature cloud significantly, correspondingly the maximum of the increasing rain on the ground increases by $2 \mathrm{~mm}$. The frontal echo is weaker, so that the region of the decreasing rainfall enlarges to the north, and the accumulated increasing rainfall in the operation area starts to decline. At 0:00 the maximum of the increasing rainfall reaches $3.5 \mathrm{~mm}$, the distribution of the increasing rainfall present South-North, and the main region of the increasing rain locates the center and south part of the heavy rain. 


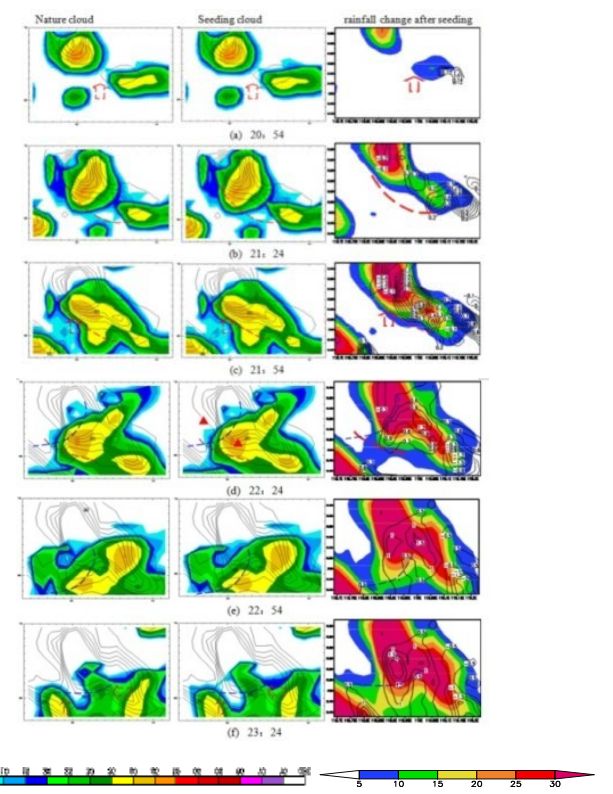

FIGURE I . THE CONTRAST VARIATION OF THE RADAR REFLECTIVITY([DBZ],SHADED) OF THE SIMULATED

NATURAL(THE FIRST COLUMN) AND SEEDING(THE SECOND COLUMN) CLOUDS AT SEEDING HEIGHT(THE ISOLINE IS INSTEAD OF THE RAINFALL ACCUMULATION, THE MINIMUM IS 5MM, THE INTERVAL IS 5MM) AND THE VARIATION OF THE DISTRIBUTION OF THE ACCUMULATIVE INCREASING RAINFALL ([MM], ISOLINE, THE THIRD COLUMN) (THE SHADE IS INSTEAD OF THE RAINFALL ACCUMULATION, THE MINIMUM IS 5MM, THE INTERVAL IS 5MM) IN THE OPERATION AREA AFTER SEEDING.

The comparison of the echo reflectivity and the accumulated rainfall between the nature and seeding cloud after seeding show that the cloud develops and strengthens and the rainfall increases after seeding AgI by rocket.

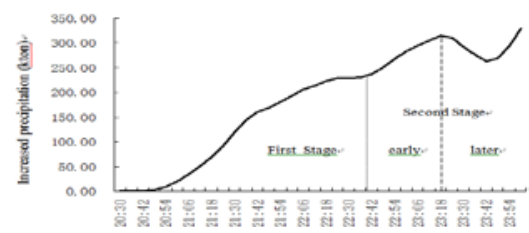

FIGURE II . THE VARIATION OF THE SIMULATED ACCUMULATIVE INCREASE OF THE RAINFALL (KTON) IN THE OPERATION AREA AFTER SEEDING WITH TIME.

\section{B. Analysis of the Microphysical Process}
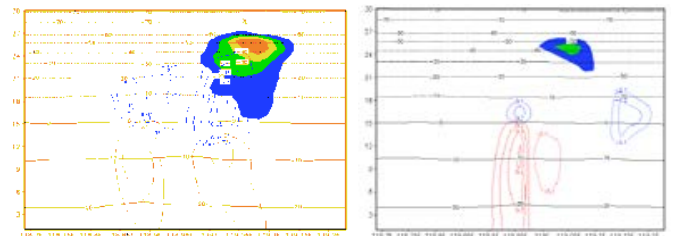

FIGURE III. THE LONGITUDE-ALTITUDE CROSS SECTION OF THE DIFFERENCE OF WATER SUBSTANCE CONTENTS (G/KG) BETWEEN THE SEEDING AND NATURAL CLOUDS (THE BLACK LINE IS INSTEAD OF THE TEMPERATURE, THE SHADE IS INSTEAD OF THE DIFFERENCE OF SNOW, THE BLUE LINE IS INSTEAD OF THE DIFFERENCE OF GRAUPLE, THE RED LINE IS INSTEAD OF THE DIFFERENCE OF RAIN) IN THE OPERATION AREA AT 21:54 AND 23:24 AFTER SEEDING.
The change of the accumulated rainfall in the operation region is divided into two phases. The longitude-altitude cross section of the difference of water substance contents between the seeding and natural clouds at the two phases(Figure3, the atmosphere is divided into high level and low level at the critical of the $0^{\circ} \mathrm{C}$ level) show that at 21:54 at the first phase after seeding there are two increasing regions of the grauple content in the upper, the maximum are $0.15 \mathrm{~g} / \mathrm{kg}$ and $0.25 \mathrm{~g} / \mathrm{kg}$ separately, and the latter overlaps the region of the snow increase. There are two regions of the rain increase; the maximum is $0.1 \mathrm{~g} / \mathrm{kg}$ in the lower, correspondingly to the two regions of the grauple increase. Meanwhile, at 23:24 at the second phase there is a region of the rain increase in the lower; maximum of $0.4 \mathrm{~g} / \mathrm{kg}$ corresponds to the region of the grauple increase in the upper, maximum of $0.2 \mathrm{~g} / \mathrm{kg}$. The contrast analysis of the water content between the upper and the lower shows that after seeding the content of the ice in the upper increases, which advances in the density of the snow and grauple. The large ice particles from the increasing snow and grauple fall into the water region in the lower and produce a great amount of the large cloud drop, so that the amount of the collection of the cloud water and rain increase, the rain in the lower increases significant and the rainfall on the ground increases.

\section{CONCLUSION}

The 3-D convectional cloud model was applied to numerical evaluation of artificial enhancingprecipitation of convective cloud in nation by now. However, the cloud model could not simulate the real atmosphere exactly due to the unreasonable boundary and initial conditions. Therefore, in test according to the mechanism of the AgI seeding and function, the AgI seeding course was coupled into the operational mesoscale WRF mode, whose boundary and initial conditions are reasonable. Then the model is applied to analyze convective precipitation enhancement over the Tian Mu Lake. The Results from analysis of real data and numerical simulation show that the model coupled with the AgI seeding course successfully simulates the convective system influencing the Tai Mu Lake region at Li Yang at night on August 28th in 2007. After analysis we find that after seeding the strong echo area of cloud enlarges, the average echo intention increases and the precipitation on the ground increases. The microphysical analysis show that after seeding the content of the ice, snow and grauple increases, correspondingly the content of the rain water in the low-level increases as well, which results in the enhancement of the precipitation over the Tian Mu Lake.

The primary application of the WRF model coupled with the AgI course in the evaluation of the artificial enhancing precipitation provides a new method for the forecast of the appropriateseeding height, seeding amount and effective precipitation enhancement.

\section{ACKNOWLEDGEMENT}

Y.W. Chen is the corresponding author. She makes the great contribution in this research.This research was financially supported by the Jiang Su MeteorologyResearch Foundation (Grant Nos. KM201403. KM201107. Q201205) . 


\section{REFERENCES}

[1] Eirh-Yu Hsie, Richard D. Farley, and Harold D. Orville, Numerical Simulation of Ice-Phase Convective Cloud Seeding,Journal of Applied Meteorology, American Meteorology Society, 19, 950-977,1980.

[2] Harold D. Orville and Fred J. Kopp, Numerical Simulation of the Life History of a Hailstorm. Journal of the Atmospheric Sciences, American Meteorology Society, 34, 1596-1618, 1977.

[3] Gregory Thompson, Roy M. Rasmussen, and Kevin Manning, Explicit Forecasts of Winter Precipitation Using an Improved Bulk Microphysics Scheme. Part I: Description and Sensitivity Analysis, Monthly Weather Review, American Meteorology Society, 132, 519-542, 2004. 